\title{
Research on Improving the Manufacturing of Titanium Medical Implants, by SLM
}

\author{
Cosma Sorin Cosmin ${ }^{1}$, Leordan Dan ${ }^{1}$ \\ ${ }^{1}$ Technical University of Cluj-Napoca, Department of Manufacturing Engineering
}

\begin{abstract}
The purpose of this study was to improve the design and manufacturing process of new medical implants, by selective laser melting. After manufacturing process, the implants were measured, microscopically and mechanical analyzed. Implants fabricated by SLM can be an attractive option for surface coatings to improve the osseointegration process. SLM process change the way that the surgeons are planning surgeries and engineers are designing custom implants. This process eliminated the constraints of shape and mechanical characteristics for implants.
\end{abstract}

Keywords: selective laser melting, process parameters, titanium, mechanical characteristics, roughness, customized implants

\section{Introduction}

The technological evolution contributed at a high pace to the continuous modernization of the medical field by improving the quality of the products and that of the medical act. The clinical practice demonstrated that titanium's biocompatibility in the organism leads to a reduced rejection rate, and the fact that the implants operate through bioresorbable or biodegradable materials coverage may intensify the formation of new tissues at the bone-implants interface, and transform them from bioinert to bioactive implants. The currently merchandised implants do not cover the multitude of the medical cases of the individual bone defects, that appeared due to some traumatisms and osteotomies required because of malformations or bones' tumor ${ }^{[1]}$. In these cases, the autografts (taken from another bone part of the patient) are commonly used. Their purpose is the replacement of a damaged bone area through an opened surgical procedure. In this type of surgery, the intraoperative challenge is given by the autograft's tough fusing process that has to be done precisely to the area of the bone's defect. In order to support the surgeons in this special kind of situations, before the surgery, starting from CT images, a pattern suitable to the affected bone area is designed, and through the selective laser melting process (SLM) a personalized implant is produced and then inserted ${ }^{[1]}$. The quick prototyping SLM is an unconventional method of manufacturing through which pieces with complex forms are made of powder, where after an initial programming, every layer of the guide mark is scanned by a solid laser.

The advantages of the manufacturing implants done with SLM technology are: the precise adjustment of the surgical site region by eliminating the shape restrictions, reducing the surgery's time by preoperative computer simulation and follow the mechanical characteristics standards. All these aspects lead to the decrease of the rejection chances, as well as to a faster recovery of the patient. In the past years, by using SLM equipment there were produced personalized implants applied in medical surgeries for the complex 
reconstruction of the bones that were up until then unsolvable medical cases and due to this fact they were an international premiere. It is about the complete replacement of the mandible with an implant that has screwed bores for the dental structure ${ }^{[2]}$ (figure 1), the replacement of the damaged zygomatic bone with a healthy symmetrical implant [3] (figure 2), total replacement arthroplasty with a prosthesis that has a lattice femoral component [4] (figure 3), the treatment for osteosarcoma located in the calcaneus through a macroporous implant [5] (figure 4) and cervical spine truss system ("Cage" implant, figure 5) ${ }^{[6]}$. The results of these surgeries are reasonable, the implants combined perfectly, without any sign of rejection, and they contributed to the improvement of the patients' health and their reunion with the community. Also in this chapter there is a study on dental implants and an analysis of the SLM process.

Surface treatments applied on titanium (Ti) implants improve the secondary containment of the endosseous implant by changing the surface topography. It has been demonstrated that the rough surfaces definitely increase the cellular and molecular mechanisms, influencing the absorption of the biomolecules from the biological fluids ${ }^{[7]}$. In vivo tests prove the fact that the treat surfaces can generate a better and faster osseointegration, in comparison with the even surfaces. From a clinical point of view, several studies have reported that on a long term the failure rate is lower in the case of the rough implants because they ensure the adhesion, the osteoblast cells proliferation and differentiation at the metal-bone interface $^{[7,8,9,10,11]}$.

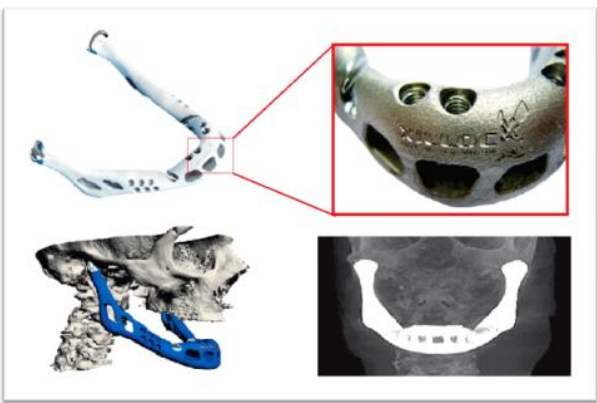

Fig. 1 Complete replacement of the mandible with an implant manufactured by SLM ${ }^{[2]}$
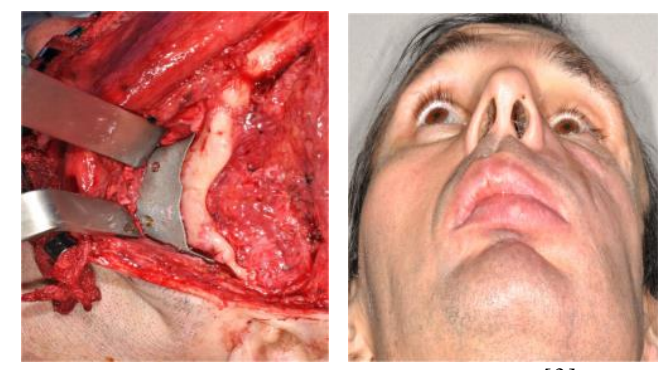

Fig. 2 Zygomatic implant ${ }^{[3]}$
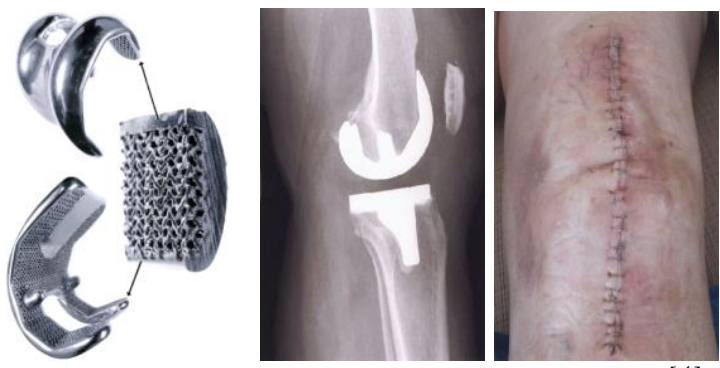

Fig. 3 Total replacement arthroplasty ${ }^{\text {[4] }}$
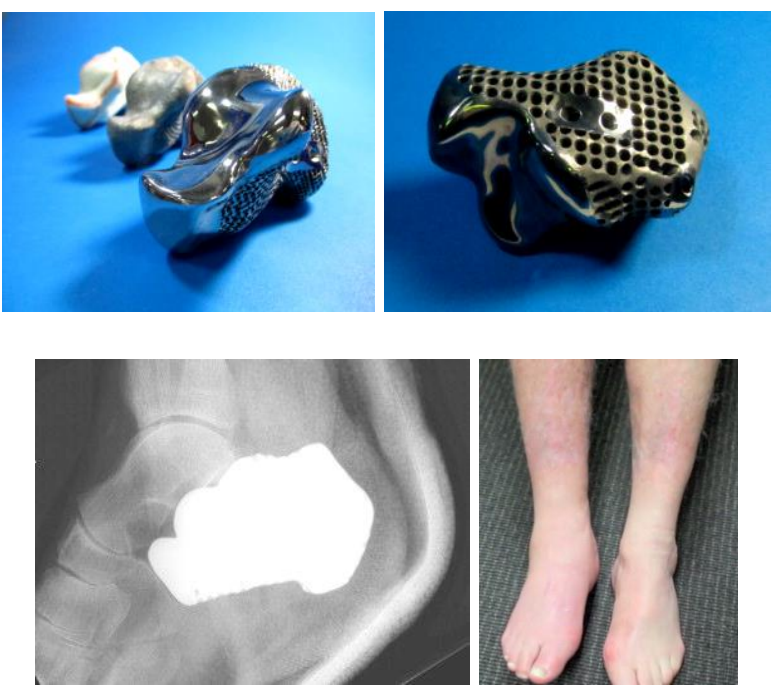

Fig.4 Porous implant for osteosarcoma treatment ${ }^{[5]}$ 

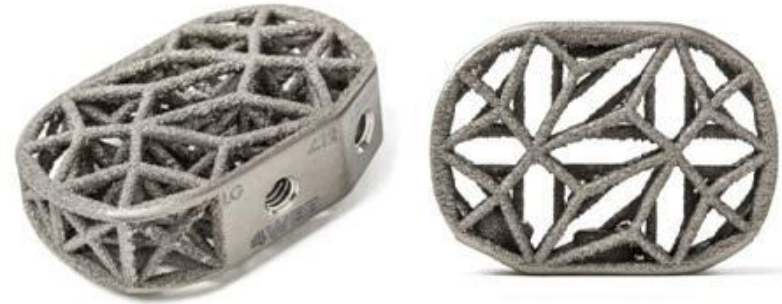

Fig. 5 Cervical spine truss system [6]

In order to obtain originality the developed research had as main objectives: the study and the current state synthesis of the SLM manufacturing domain through medical implants from $\mathrm{Ti}$, the development of a new configuration method of the SLM process parameters that will improve the mechanical characteristics of the implants, the increase of the surface quality and the decrease of the dimensional deviations, the functioning of the manufactured implants, the testing of the theoretical contributions and of the new processing SLM method through experimental research and case studies on the manufacturing of personalized implants.

\section{Materials and Methods}

Pure titanium powder (TILOP 45) has $45 \mu \mathrm{m}$ particle diameter, the melting point temperature around $1670^{\circ} \mathrm{C}$ and the following chemical constituents: $99.7 \% \mathrm{Ti}, 0.08 \% \mathrm{Fe}$, $0.17 \% \mathrm{O}, 0.03 \% \mathrm{C}, 0.03 \% \mathrm{~N}, 0.01 \% \mathrm{H}$.

SLM ReaLizer 250 system (MTT Technologies, Germany) uses selective laser melting technology, an additive manufacturing process. All the parts were manufactured with this equipment.

\section{Case study - Customized implants fabricated by SLM process}

Previous research has presented the difficulties that appear during the time of the SLM process, so it is imposed a new approach of the manufacturing method for limiting the residual tension stored by the pieces during processing $[9,12]$. The new programming method of the Hatch function responsible for scanning the sections it was set by reducing to half the laser's exposure time (ET) and the distance between the points (PD), having at the end the same scanning speed. Through this approach of the process parameters was achieved a reduction of the inner tension between the pieces that appears during the SLM manufacturing time, making possible to execute parts with complex shapes (figures 6 and 7).

The configuration of the process parameters in this way required a powerpropelled testing of some standard samples manufactured under these conditions. For determining the mechanical characteristics 6 sets of samples were produced with the laser power (P) between 120-160 W and $400 \mathrm{~mm} / \mathrm{s}$ or $1000 \mathrm{~mm} / \mathrm{s}$ scanning speed. The direction of the traction force was the same as the structure of the scanned layers, and the tests' results were expressed in tablets and conventional characteristic curves. The improved mechanical characteristics of the manufactured marks made through this method distinguish themselves, obtaining with some process parameters (ET $100 \mu \mathrm{s}$, PD $40 \mu \mathrm{m}, \mathrm{P} 120 \mathrm{~W}$ ) uniform and ductile pieces with mechanical properties within the standard surgical implants ASTM F76 (441 MPa ultimate tensile strength, $420 \mathrm{MPa}$ yield tensile strength, $101 \mathrm{GPa}$ Young modulus).

The double scan with $160 \mathrm{~W}$ and $1000 \mathrm{~mm} / \mathrm{s}$ leads to the reduction of the stiffness of the endosseous implants from $\mathrm{Ti}$ that directly influences the cell receptors and the focal points, initiating a burst of intercellular signals that determine a specific genetic activation. In these process' conditions the elastic longitudinal modulus of the grafting from $\mathrm{Ti}$ can be reduced by $60 \%$ until $41 \mathrm{GPa}$ (cortical bone 3-25 GPa), without compromising the ultimate tensile strength, that in this case was $508 \mathrm{MPa}$ being the peak value achieved in all mechanical attempts.

The modification of the Ti's physic-mechanical characteristics through 
SLM process parameters presents new opportunities in medicine field because there can be manufactured endosseous grafts with mechanical properties interrelated with those of the host osseous tissue, taking into account the patient age, sex and health condition, by offering a high grade of biocompatibility and reducing the risk of rejection.

Knowing that the secondary containment of the dental implants is directly influenced by the quality of the surfaces, and the pieces manufactured through SLM showed initially a high $R_{a}$ roughness $(\sim 10 \mu \mathrm{m}){ }^{[13]}$. The optimization of the process parameters was performed using ANOVA method, and there were defined sets of the best parameters depending on the desired roughness values. The study distinguished itself with a slight decrease under the level of other studies of the $\mathrm{R}_{\mathrm{a}}$ roughness by proper configuration of the SLM process parameters without any post-processing application techniques. Given the international medical trends, the present pieces of research contributed to the possibility of direct manufacturing of $R_{a}$ roughness implants between 3-4 $\mu \mathrm{m}$. This roughness is considered by doctors to represent the ideal surface that directly influences the mechanical fixation of the new bone tissue to the implant ${ }^{[11]}$. By reducing the roughness of the manufactured SLM pieces there will be a leading to the optimization of the post-processing methods, and the correlation of the process parameters (associated with the scanning of the outline) with the amount of the laser's adjustment beam, it reduces the dimensional deviation from $\pm 0,1 \mathrm{~mm}$ to $\pm 0,05 \mathrm{~mm}$.

The dental implants are merchandised with standard options (length, diameter, screw) that sometimes do not match the patient's individual requirements, aspects that were revealed by numerous researches ${ }^{[14]}$. In figure 6 there are presented three SLM prototype implants projected and manufactured with the purpose of developing the personalized endosseous implants system.

a)

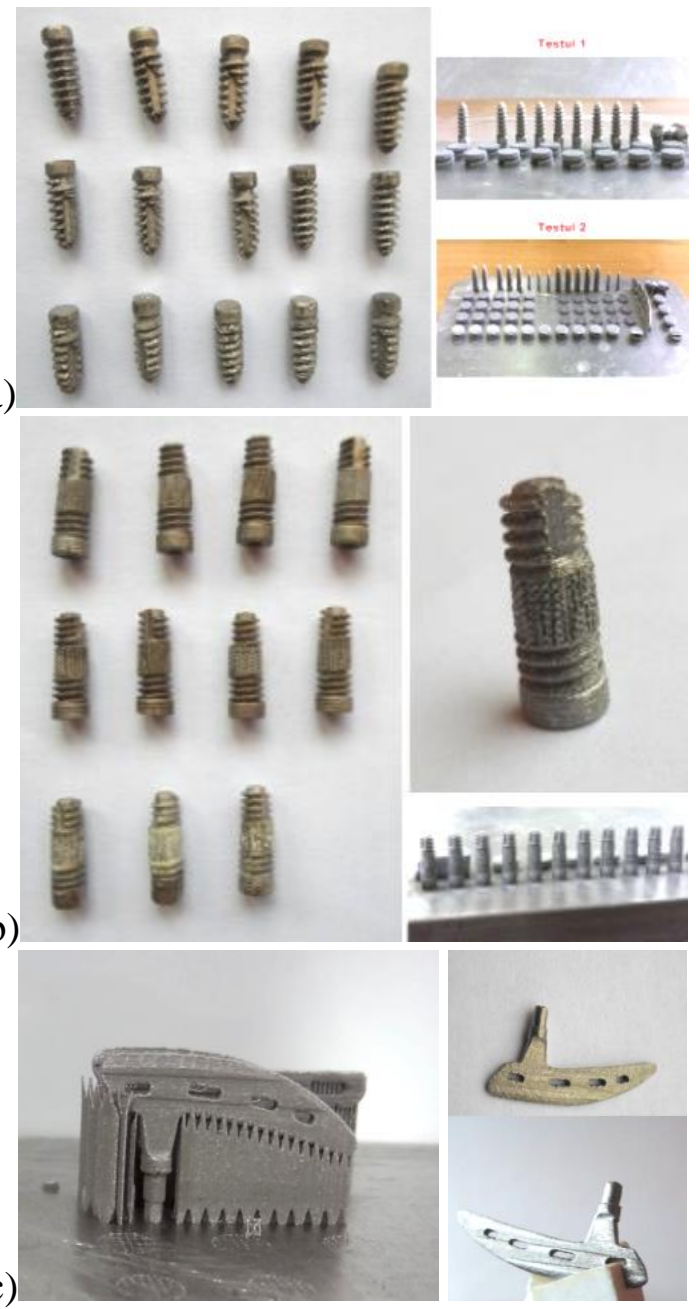

Fig. 6 Prototype dental implants manufactured by SLM: a) Compact implant screw,

\section{b) Implant with macropous zone, c) Blade implant}

In order to transfer the concept of the lattice structures from experimental marks into medical applications, one set of dental implants had a median macroporous area, defined by a specific programming function Point Structure (it is not CAD designed). The vertical orientation work platform of the CAD patterns and the designed marks made only on the upper surface of the collar made possible the manufacturing of the 33 implants by 
adapting the SLM process parameters. The interconnected pore sizes were manufactured between $0,21-0,53 \mathrm{~mm}$, and by overlaying this information data over that from the medical field, it can be assumed that the lattice structures will support the development of the new bone tissues with blood vessels, that will reduce the rejection risk of the implant with such a microporosity ${ }^{[15,16]}$. The manufactured dental implants were tested on bovine bone with the purpose of checking the resistance of the screw thread and the possibility of exfoliation when inserted. These implants were applied in osteotomies with a couple of high torsion $\left(100 \mathrm{~N}^{*} \mathrm{~cm}\right)$ because the structure of the screw presented deformation of shape and irregular craters, aspects highlighted after the SEM microscopy analysis [14]. This moment of distortion in does not correspond to the dental domain because it can compromise the host bone and damage the osseointegration ${ }^{[17]}$. It is necessary to diminish it by an additional processing of the screw through carving. Both the implants with firm screw and those with macroporosity area resisted to the forced insertion in undersized osteotomies without exfoliating.

In addition, the paper summarizes the results of some experimental research developed for the systematization of the SLM process where different experimental implants were manufactured (figure 7).

a)

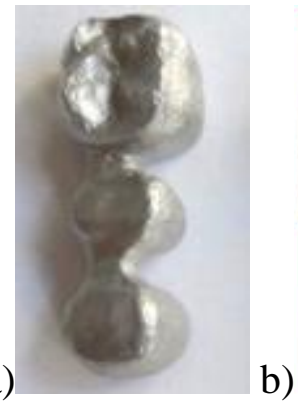

Fig. 7 a) Dental bridges, b) Macroporous grafts

\section{Conclusions}

The mechanical properties of Ti can be adapted to the requirements of patients to be friendlier. Using the proper process parameters, the mechanical characteristic of implants manufactured by SLM could respect the conditions required in ASTM F67 (Standard Specification for Unalloyed Titanium, for Surgical Implant Applications).

Without any post-processing methods, the parts manufactured in optimal conditions have the roughness $R_{a}$ between 3-4 $\mu \mathrm{m}$. From a technological point of view, SLM manufacturing prototype blade implants is possible, and their coverage with apatite through SLA method could lead to their temporal usage in implantology satisfying some complex medical needs.

The SLM manufactured implants and custom devices used in medicine might lead to the development of some therapeutic acts in some malformation disorders unsolved through classic procedures. The methodology followed in CAD design, FEA simulation, SLM manufacturing of customized implants, offered new opportunities in medicine.

Further researches will include in vivo test, to check the osseointegration process on these microporous implants fabricate by SLM.

\section{Acknowledgment}

This research was supported by the AM-CIR project, PN-II-RU-TE-2014-4-1157, no. 37/01.10.2015 financed from the UEFISCDI by the Romanian Government.

This paper was also supported by the

Sectoral Operational Programme Human Resources Development POSDRU/159/1.5/S/137070 financed from the European Social Fund.

\section{References}

1. S.C. Cosma, S. Matei, C. Vilau, Medical manufacturing innovations, Balneo Research Journal, Vol.6 (1), 2015. 
2. I. Kroonenburgh, I. Lambrichts, J. Poukens, Doctor and engineer creating the future for 3D printed custom made implants, Digital Dental News, Pp. 60-65, 2012.

3. H. Rotaru, R. Schumacher, S. Kim, C. Dinu, Selective laser melted titanium implants: a new technique for the reconstruction of extensive zygomatic complex defects, Maxillofacial Plastic and Reconstructive Surgery, 37(1): 1, 2015.

4. L.E. Murr, S.M. Gaytan, E. Martinez, F. Medina, R.B. Wicker, Next Generation Orthopaedic Implants by Additive Manufacturing Using Electron Beam Melting, International Journal of Biomaterials, Article ID 245727, 2012.

5. J. Imanishi, P.F. Choong, Three-dimensional printed calcaneal prosthesis following total calcanectomy, Int J Surg Case Rep, 10: 83-87, 2015.

6. http://4webmedical.com/

7. S.C. Cosma, N. Balc, M. Moldovan, C. Borzan, Surface Treatments Applied on Titanium Implants, Ovidius University Annals of Chemistry, 26(1):41-48, 2015.

8. J. E. Biemond, G. Hannink, N. Verdonschot, P. Buma, Bone ingrowth potential of electron beam and selective laser melting produced trabecular-like implant surfaces with and without a biomimetic coating,

J Mater Sci: Mater Med, 24: 745-753, 2013.

9. S.C. Cosma, N. Balc, D. Leordean, M. Moldovan, M. Dudescu, C. Borzan, Customized Medical Applications of Selective Laser Melting Manufacturing, Academic Journal of Manufacturing Engineering, 13(1), 2015.

10. A. S. Azevedo, M. J. Sa, M.V. Fook, P.I. No'brega Neto, O.B. Sousa, S. Azevedo, M.W. Teixeira, F.S. Costa, A.L. Araujo, Use of chitosan and $\beta$-tricalcium phosphate, alone and în combination, for bone healing în rabbits,
J Mater Sci: Mater Med, 25: 481-486, 2014.

11. M. Herrero-Climent, P. Lázaro, J. Vicente Rios, S. Lluch, M. Marqués, J. Guillem-Martí, F. Gil, Influence of acid-etching after grit-blasted on osseointegration of titanium dental implants: in vitro and in vivo studies, J Mater Sci Mater Med., 24(8): 2047-55, 2013.

12.S.C. Cosma, A. Miron, A. Radu, Zygomatic Implants Manufactured by SLM, Acta Technica Napocensis, 58(2):251-256, 2015.

13. N. Balc, S.C. Cosma, J. Kessler, V. Mager, Research on improving the outer surface quality of the parts made by SLM, Applied Mechanics and Materials, Vol. Modern Technologies in Manufacturing, Pp. 193-198, 2015.

14. S.C. Cosma, N. Balc, D. Leordean, S. Matei, Dental Implants with Lattice Structure Fabricated by Selective Laser Melting, International Virtual Research Conference in Technical Disciplines, Zilina, Slovakia, 2014.

15. A. Nouri, P. D. Hodgson, C. Wen, Biomimetic Porous Titanium Scaffolds for Orthopedic and Dental Applications, Biomimetics Learning from Nature, Ed. Amitava Mukherjee - InTech, 2010.

16. M. Jasty, H. Kienapfel, P. Griss, Fixation by Ingrowth, Ed. Lippincott Williams and Wilkins, Philadelphia, 2007.

17. M.M. Goswami, Mukul Kumar, Abhinav Vats, A.S. Bansal, Evaluation of dental implant insertion torque using a manual ratchet, Medical Journal Armed Forces India, 2013. 